\title{
Doxorubicin Hydrochloride Loaded Polyanhydride Nanoformulations and Cytotoxicity
}

\author{
Nagaraja Sreeharsha ${ }^{1,2 *}$, Jagadeesh G Hiremath ${ }^{3}$, Prashanth Kumar $\mathbf{R}^{4}$, Girish Meravanige ${ }^{5}$, \\ Saleemulla Khan ${ }^{6}$, Ranjith Kumar Karnati7, Mahesh Attimarad', Bandar Al-Dhubiab', Anroop \\ Balachandran Nair ${ }^{1}$, Katharigatta Narayanaswamy Venugopala ${ }^{1,8}$
}

${ }^{1}$ Department of Pharmaceutical Sciences, College of Clinical Pharmacy, King Faisal University, Al-Ahsa, SAUDI ARABIA. ${ }^{2}$ Department of Pharmaceutics, Vidya Siri College of Pharmacy, Off Sarjapura Road, Bangalore, Karnataka, INDIA. ${ }^{3}$ Department of Pharmaceutics, PA College of Pharmacy, Mangalore, Karnataka, India.

${ }^{4}$ Department of Pharmaceutics, East West College of Pharmacy, Bangalore, Karnataka, INDIA.

${ }^{5}$ Department of Biomedical Sciences, College of Medicine, King Faisal University, Al-Ahsa, SAUDI ARABIA.

${ }^{6}$ Department of Pharmacognosy, PA College of Pharmacy, Mangalore, Karnataka, INDIA.

${ }^{7}$ Department of Chemistry, College of Science, King Faisal University, Hofuf, SAUDI ARABIA.

${ }^{8}$ Department of Biotechnology and Food Technology, Durban University of Technology, Durban, SOUTH AFRICA.

\begin{abstract}
This study aimed to evaluate DOX.HCl loaded P(DLLA-CO:60:40) nanoformulations with a copolymer of $\mathrm{F} 127$ as a potential drug delivery system for cancer. $\mathrm{DOX} \cdot \mathrm{HCl}$ was encapsulated in nanoformulations of Poly DL-lactic acid co castor oil 60:40, P(DLLACO:60:40) polymer with the copolymer/stabilizer Pluronic ${ }^{\oplus}$ F127. The mean diameter of the cylindrical rod shape of the nanoformulation particles was $248 \pm 2.4-260 \pm 3.2 \mathrm{~nm}$ with an acceptable poly dispersibility of $0.29-0.33$ and a smooth surface as visualized by DSC, SEM and XRD displayed that DOX. $\mathrm{HCl}$ was present as an disordered crystalline or amorphous state in the nanoformulations. The nanoformulations showed a steady pattern of drug discharge for $24 \mathrm{hrs}$. F-3 and F-4 formulations had IC ${ }_{50}$ values of $3.2 \pm 0.03$ and $1.98 \pm 0.08 \mathrm{mcg} / \mathrm{ml}$ while the free drug inhibition concentration of $\mathrm{IC}_{50}$ was $2.2 \mathrm{mcg} / \mathrm{ml}$. The drug-loaded nanoformulations showed significant cytotoxic effects on MCF-7 breast cancer cell lines.
\end{abstract}

Key words: Doxorubicin hydrochloride, MCF-7 breast cancer cell lines, Nanoformulations, Drug delivery.

\section{INTRODUCTION}

Preparation of pharmaceutically sustained or controlled drug delivery systems with polyanhydride blend polyester polymers has been explored extensively for their biodegradation and biocompatibility. Most recent investigations testified to the efficiency of an influenza A virus (IAV) subunit vaccine based on a biodegradable polyanhydride nanoparticle delivery system. ${ }^{1-6}$ In the natural form polyanhydrides constitute a hydrophobic backbone linked with hydrophilic anhydride. The different analysis groups in vitro and in vivo showed that the composition of polymer or monomers could control biodegradation; biodegradable products of carboxylic acids are non-toxic and non-mutagenic. The ring-opening polymerization, melt polycondensation and interfacial condensation methods are employed to produce polyanhydrides. ${ }^{7-9}$ Domb et al. described the synthesis of the biodegradable polymer composites of poly(hydroxybutyrate) (PHB), polysebecic anhydride (PSA) and polyesters of Poly (lactic acid) (PLA) and poly(caprolactone) (PCL). In brief, the different blends show different properties compared with the corresponding parent polymers; the content of rapidly degrading PSA containing polymeric blends of polyesters was the ratedetermining step for the release of drugs. ${ }^{4-5}$ In previous studies they investigated how
Submission Date: 14-07-2020; Revision Date: 08-08-2020; Accepted Date: 01-10-2020

DOI: 10.5530/ijper.55.1.2 Correspondence: Nagaraja Sreeharsha Department of

Pharmaceutical Sciences, College of Clinical

Pharmacy,King Faisal University, Al-Ahsa, SAUDI ARABIA.

Phone: +96-653-548-5322 E-mail: sharsha@kfu.edu.sa

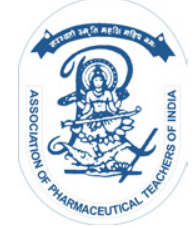

www.ijper.org 
lactide and glycolide polymers undergo bulk hydrolysis and the sequence of erosion and diffusion of drug release; to diminish problems related to erosion and diffusion they incorporated polyols to diketeneacetals and specifically added a latent acid segment in the polymer backbone to influence the erosion process. ${ }^{10-13}$ PLA is one of the best biomaterials with few limitations with its hydrolytic release process. The complete degradation of PLA in the body occurs in a few weeks to months, with bulk erosion showing non-linear drug release, the chance of unreleased drug in an acidic environment, and the discontinuous release of drugs from PLA carriers due to bulk erosion or surface erosion of the polymer. The purpose of the PLA polymer in injections and aerosol preparations was to overcome the pronounced agglomeration of PLA particles that makes it difficult to suspend particles. To overcome the role that low or high molecular weight plays in drug release from polyester derivatives, and based on the hydrophobicity or hydrophilicity requirements of the polymer, the ideal molecular weight of the polyester are synthesized with the anhydride (polyanhydride) linkages to enhance the controlled release of drug. ${ }^{11,13}$ Later studies described the release aspects of mixed polyester blend with polyanhydride polymers. These polymers comprised of hydroxy ended prepolymer, acid-ended prepolymer linked with hydrolytic anhydride leads to an increasing rate of surface erosion; erosion was biphasic and the faster release of polyester components with slower degradation of polyester components. ${ }^{13}$ In another example, polyesters are merged with ricinoleic acid (hydroxy fatty acid) of castor oil components of polyester/anhydride polymers for drug carrier systems. The castor oil (CO) comprises a cis-double bond that can be hydrogenated, halogenated, polymerized and oxidized. The ricinoleic acid leads to high hydrophobicity of the polyesters, changing the mechanical and physical properties of polymers." Our research group investigated the use of these polymers with paclitaxel; tamoxifen citrate loaded sustained/controlled drug delivery systems using polyester blends of polyanhydride. ${ }^{14-17}$ The particle shape and surface morphology are significant in the successful improvement of nanoformulations. Manipulation of the shape and surface can contribute considerably to the interaction with living media since particle shape and constitutional structure affect cellular uptake, toxicity and in vivo target of the drugloaded carriers. ${ }^{18}$ Smooth surface particles, including onions, snowmen, dumbbells, rattles, cylindrical and branched particles such as rods, stars, flowers and urchins particles showed potential applications in drug delivery systems. ${ }^{19-21}$ Our present research is aimed at preparation of doxorubicin hydrochloride $(\mathrm{DOX} \cdot \mathrm{HCl})$ loaded poly (dl-lactic acid co-castrol oil 60:40 P (DLLA:CO:60:40) nanoformulations. $\mathrm{DOX} \cdot \mathrm{HCl}$ is a chemotherapeutic used to treat many cancers. DOX inhibits the topoisomerase II complex and a major response mechanism is production of reactive oxygen species which is involved in cell necrosis and apoptosis. In clinical studies $\mathrm{DOX} \cdot \mathrm{HCl}$ showed significant cytotoxicity against malignant cells; there are dosedependent toxicities such as myelosuppression and cardiotoxicity. ${ }^{21,22}$ Therefore, a different drug carrier is needed to reduce the dose and side effects. DOX $\cdot \mathrm{HCl}$ encapsulated in hydrophilic (DL lactic acid) and hydrophobic castor oil, based P-(DLLA-CO:60:40) with a copolymer of poloxamer, Pluronic ${ }^{\mathbb{B}} \mathrm{F} 127$ (PF127, PEO-PPO-PEO in amphiphilic triblock copolymer could be an excellent successor to current formulations. This encapsulation would provide hydrophilicity to the $\mathrm{DOX} \cdot \mathrm{HCl}$ polymeric nanoformulations for sustained or controlled release of the drug with time.

\section{EXPERIMENTAL}

\section{Materials}

Poly DL: Lactic acid co-castor oil 60:40, referred to as P(DLLA-CO:60:40) was received as a gift from the School of Pharmacy, Hebrew University of Jerusalem, Israel. The P (DLLA-CO:60:40) molecular weight ( $\mathrm{mw}$ ) was 5576 , and the monomer number was 4963; DOX $\cdot \mathrm{HCl}$ was obtained from RPG Life Sciences Ltd, Mumbai. F127 (PF-127, PEO-PPO-PEO block copolymer, an amphiphilic triblock copolymer, and DL lactic acid LA and castor oil, Human breast cancer cell lines (MCF-7) were purchased from the National Centre for Cell Sciences (Pune, India), (CO) were procured from Sigma-Aldrich Corporation (St Louis, MO, USA). All other chemical reagents were analytical grade.

\section{UV-Visible Spectrophotometric analysis for DOXHCL}

The DOX $\cdot \mathrm{HCl}$ content in the nanoformulations was detected using a modification of a published UV-Visible spectrophotometric method. ${ }^{21}$ Briefly, $10 \mathrm{mg}$ of DOX $\cdot \mathrm{HCl}$ was dissolved in $100 \mathrm{~mL}$ phosphate-buffered saline (PBS, $\mathrm{pH}$ 7.4); from this $100 \mathrm{mcg} / \mathrm{mL}$ was made with PBS, and this stock solution was filtered through a $0.22 \mu \mathrm{m}$ filter (Millipore, India). The stock solution was scanned over a wavelength range of $800-200 \mathrm{~nm}$ against PBS as a blank with double beam Shimadzu UV-1800 UV-VIS-spectrophotometer. The spectrum 
of absorbance versus wavelength was recorded. The spectrum was determined for the absorbance maximum $\left(\lambda_{\max }\right)$, and the highest absorbance maximum of $482.0 \mathrm{~nm}$ was selected as the $\lambda_{\text {max }}$ for further readings $\mathrm{DOX} \cdot \mathrm{HCl}$. DOX $\cdot \mathrm{HCl}$ was diluted serially from $2-20$ $\mathrm{mcg} / \mathrm{ml}$. The absorbance results were assessed at the $\lambda_{\max }$ against PBS as a blank. The plot of absorbance vs. drug concentration $(\mu \mathrm{g} / \mathrm{ml})$ was plotted, the data subjected to linear regression, and the intercept and regression coefficient were estimated using Microsoft Excel (2007).

\section{Preparation of DOXHCL loaded (P(DLLA:CO: 60:40) nanoformulations}

The nanoformulations of $\mathrm{DOX} \cdot \mathrm{HCl}$ containing P-(DLLA-CO:60:40) polymers were developed by a modified solvent evaporation thin-film hydration technique as previously reported. ${ }^{23-25}$ Four different drug concentrations with loading nanoformulations of $\mathrm{DOX} \cdot \mathrm{HCl}$, Pluronic ${ }^{\circledR} \mathrm{F}-127$ and polymer were dissolved in $15 \mathrm{ml}$ of dichloromethane, which was evaporated in a vacuum rotary flash evaporator to form a thin film. The resulting velvety film was hydrated with $30 \mathrm{ml}$ of $10 \mathrm{nM}$ HEPES buffer $\left(\mathrm{pH} 7.4,37^{\circ} \mathrm{C}, 4\right.$ hrs). The suspension was homogenized at $500 \mathrm{rpm}$ for eight minutes, and the mixture subjected to ultrasound waves (10 mins, 80 kilo coulombs (KC80), 80 watts (w) to form a nanoformulation. The formulation was centrifuged (10000 rpm, $\left.4^{\circ} \mathrm{C}, 10 \mathrm{mins}\right)$, and rinsed 3-4 times with double distilled water to remove free DOX and excipients. These dimented nanoparticles were resuspended in $30 \mathrm{ml}$ of pure de-ionized water (D.I.) and freeze dried(-60 $24 \mathrm{hrs}$ ) with antifreezing substances (8\% w/w of polymers and drug). DOX $\cdot \mathrm{HCl}$ free blank P-(DLLA-CO:60:40) nanoformulations were prepared by the same method. The formulation composition is shown in Table 1.

\section{Characterization of nanoformulations}

The lyophilized nanoformulations were weighed, and the percentage yield was determined by equation (1). The percentage entrapment efficiency of $\mathrm{DOXHCl}$ loaded nanoformulations (Table 2) was determined as follows: the nanoformulations were suspended in 10 $\mathrm{ml}$ PBS ( $\mathrm{pH}=7.4)$, centrifuged (Remi Pvt Ltd, India) (12000 rpm, $4^{\circ} \mathrm{C}, 10 \mathrm{mins}$ ), and the supernatant was collected and diluted to $2-20 \mathrm{mcg} / \mathrm{ml}$. The DOXHCl content was analyzed by UV-Visible spectrophotometry, and the entrapment efficiency (EE) was estimated by equation (2). The percentage of loading was determined by equation (3).
(1) $\%$ yield $=$ amount of nanoformulations obtained/ amount of the drug, polymer, and cryoprotectant $\times 100$ (2) $\% \mathrm{EE}=$ Total drug-drug [supernatant] / drug [total] $\times 100$

(3) Percentage of DL = amount of actual drug entrapped in nanoformulations/ total amount (drug+ excipients) $\times 100$.

We used a Brookhaven Zeta Plus light diffraction analyzer (Holtsville, NY, USA) to measure the mean particle size of the nanoformulation samples ${ }^{24}$ For analysis, the $\mathrm{DOX} \cdot \mathrm{HCl}$ nanoformulation was suspended in $10 \mathrm{ml}$ water. The suspended particles were sonicated (10 mins) and the light scattering with time at a definitive angle after interaction with particles measured. Samples were assessed at a temperature rate of $10^{\circ} \mathrm{C} / \mathrm{min}$, in the range of 10 degree Celsius to 230 degree Celsius using DSC822e differential scanning calorimeter (DSC) $)^{25-28}$ (Mettler Toledo, Polaris Parkway, USA) adopted to assess the degree of crystallinity and thermal properties.An empty pan was used as a reference. The $\mathrm{X}$-ray diffraction (XRD) of the nanoformulations was assessed with Philips PW 1820/00 automated diffractometer with $\mathrm{Cu}-\mathrm{Ka} \alpha$ radiations to measure the crystal quality of the samples. The lyophilized samples were examined by scanning electron microscopy after they had been gold sputtered (JSM-848, Joel, Japan).

\section{In vitro degradation of nanoformulations}

The $\mathrm{DOX} \cdot \mathrm{HCl}$ containing nanoformulations in vitro dissolution studies were carried out using a water bath shaker (Remi Equipment, India). Capped bottles (50 $\mathrm{mL}$ ) carrying DOX $\mathrm{HCl}$ loaded nanoformulations in 30 $\mathrm{ml}$ of phosphate buffered solution (PBS, $\mathrm{pH}=7.4$ ) as release medium were incubated at 37 for 24 hrs. The initial sample was withdrawn at 30 mins followed by sampling every hour. The samples were centrifuged (12000 rpm, 10 mins, the supernatant was collected, samples were diluted to $2-20 \mathrm{mcg} / \mathrm{mL}$ with phosphate buffered saline $(\mathrm{pH}=7.4)$ and the $\mathrm{DOX} \cdot \mathrm{HCl}$ content was analyzed as described above.

\section{In vitro cytotoxicity}

The cytotoxic efficiency of DOX $\cdot \mathrm{HCl}$ loaded P-(DLLA-CO:60:40) nanoformulations compared to blank nanoformulations as control were evaluated using the MCF-7 human breast cancer cell line. MCF-7 cells were grown in Dulbecco's modified Eagle's medium enhanced (DMEM, with 10\% fetal bovine serum (FBS)), streptomycin $(100 \mathrm{mg} / \mathrm{ml})$, amphotericin B (5 $\mathrm{mg} / \mathrm{ml}$ ) and penicillin $(100 \mathrm{IU} / \mathrm{ml})$ and in a humidified atmosphere of $5 \% \mathrm{CO}_{2}$ at $37^{\circ} \mathrm{C}$. The cell stock was prepared in $25 \mathrm{~cm}^{2}(60 \mathrm{~mL})$ culture vials and aliquots of 
$1 \mathrm{X} 10^{5}$ cells/well added to 96 well $\mathrm{V}$ bottom microtiterplates (Tarsons products [P], Ltd. Kolkata, India). The cells were grown to confluence and released from the plastic with a trypsin phosphate versene glucose (TPVG) solution $(0.02 \%$ EDTA, 1 X $0.25 \%$ trypsin, $0.05 \%$ glucose in PBS). The released MCF-7 cells were diluted to $1 \mathrm{X} 10^{5}$ cells $/$ well $/ 0.1 \mathrm{~mL}$ in DMEM-FBS. After 24 hrs, when a limited monolayer occurred, the supernatant was decanted, and the monolayer was washed twice with PBS. DOX.HCl and nanoformulation suspensions containing drug concentrations from $0.01-10 \mathrm{mcg} / \mathrm{ml}$ in $200 \mu \mathrm{L}$ were added to wells. After 24 hrs the media was removed, $50 \mu \mathrm{l}$ of MTT reagent was added to each well and incubated with shaking $\left(3 \mathrm{hrs}, 37^{\circ} \mathrm{C}\right)$. The supernatant was removed, $200 \mu$ l of propanol was added and the plates were gently shaken to solubilize the formed formazan. The absorbance was measured using a microplate reader at a wavelength of $540 \mathrm{~nm}$. The cell survival was determined as a percentage of control. ${ }^{1,29}$

\section{RESULTS AND DISCUSSION}

\section{Preparation and characterization techniques of nanoformulations}

Nanoformulations in a copolymer of F127 prepared by a thin-film hydration technique and loaded with 5 to $20 \%$ w/w DOX $\cdot H C l$ P-(DLLA-CO:60:40) produced a high yield of the blank and drug-carrying nanoformulations. This method is straight forward with no practical difficulties and generates a high yield of small, uniform particles. ${ }^{29}$ The production yield of the blank nanoformulations was $96.89 \%$ and for the DOX.HCl loaded nanoformulations (F-1-F-4) it was 96 to $98 \%$. The encapsulation of $\mathrm{DOX} \cdot \mathrm{HCl}$ with polymer and copolymer depends upon the solvent, hydration time, temperature, and F127. We use dichloromethane as a solvent since it has acceptable and limited toxicity. The hydration time was maintained for $4 \mathrm{hrs}$ (the optimal hydration time) and the temperature was maintained at 37 , resulting in high encapsulation efficiency. Due to the effect of poloxamer, the block copolymer, P(DLLA-CO:60:40) and DOX.HCl initially were dissolved in universal solvent; this is an advantageous step for the F127 since at elevated temperature it influences dynamic change in the system composition and solubilization of the drug within the main polymeric matrix resulting in high encapsulation efficiency and high drug-loading. ${ }^{2,3}$ Other studies found similar outcomes for DOX-loaded disulfide-linked polyethylene glycol lysine-di-tocopherol succinate nanomicelles. Another reason may be sufficient amount of drug/polymer ratio, thus not causing saturation of encapsulation. Almost constant drug loading efficiency was seen in this study, which might be due to a fixed amount of polymer available for drug loading. ${ }^{30-31}$ The

\begin{tabular}{|c|c|c|c|c|}
\hline \multirow[t]{2}{*}{ Formulations } & Drug & Polymer & $\begin{array}{l}\text { Drug } \\
\text { loading }\end{array}$ & Poloxamer- F127 \\
\hline & $\begin{array}{l}\text { Doxorubicin } \\
\text { (mg) }\end{array}$ & $\begin{array}{l}\text { p(DLLA:CO) } \\
60: 40(\mathrm{mg})\end{array}$ & $\%$ w/w & $\begin{array}{l}\text { Total weight of Drug and } \\
\text { Polymer }(10 \% \mathrm{w} / \mathrm{w})(\mathrm{mg})\end{array}$ \\
\hline Blank & - & 200.0 & - & 20.0 \\
\hline $\mathrm{F}-1$ & 10.0 & 200.0 & 5.0 & 21.0 \\
\hline $\mathrm{F}-2$ & 20.0 & 200.0 & 10.0 & 22.0 \\
\hline$F-3$ & 30.0 & 200.0 & 15.0 & 23.0 \\
\hline $\mathrm{F}-4$ & 40.0 & 200.0 & 20.0 & 24.0 \\
\hline
\end{tabular}

\begin{tabular}{|c|c|c|c|c|c|c|}
\hline \multicolumn{7}{|c|}{ Table 2. Characterization of DOXHCI loaded nanoformulations } \\
\hline FM & $\begin{array}{c}\text { Yield } \\
(\% \mathbf{w} / \mathbf{w})\end{array}$ & $\begin{array}{c}\text { Theoretical } \\
\text { Drug-Loading } \\
(\% \mathbf{w} / \mathbf{w})\end{array}$ & $\begin{array}{c}\text { Practical Drug } \\
\text { Loading } \\
(\% \pm S D)\end{array}$ & $\begin{array}{c}\text { Encapsulation } \\
\text { efficiency } \\
(\% \pm S D)\end{array}$ & $\begin{array}{c}\text { Average } \\
\text { Particle Size } \\
(\mathbf{n m \pm S D )}\end{array}$ & $\begin{array}{c}\text { Polydispersity } \\
\text { Index }\end{array}$ \\
\hline Blank & $\mathbf{9 6 . 8 9}$ & - & & - & $\mathbf{2 6 6 \pm 0 . 5}$ & $\mathbf{0 . 2 9}$ \\
\hline F-1 & 96.81 & 5 & 3.83 & 96.8 & $256 \pm 1.4$ & 0.31 \\
\hline F-2 & 97.91 & 10 & 7.63 & 95.2 & $248 \pm 2.4$ & 0.32 \\
\hline F-3 & 98.70 & 15 & 11.05 & 96.4 & $254 \pm 1.6$ & 0.31 \\
\hline F-4 & 97.75 & 20 & 14.23 & 96.8 & $260 \pm 2.2$ & 0.33 \\
\hline
\end{tabular}


particle size of nanoformulations of $\mathrm{DOX} \cdot \mathrm{HCl}$ loaded P(DLLA-CO:60:40) a copolymer of F127 had a mean diameter of $248 \pm 2.4$ to $260 \pm 3.2$ and had an acceptable polydispersibility of 0.29 to 0.33 , respectively. The shape and morphology of drug-loaded nanoformulations were cylindrical with a smooth surface and a good dispersibility by SEM (Figure 1). The average diameter was 130 $\mathrm{nm}$ (D). These results indicate that nanoformulation showing sizes less than 500-1000 $\mathrm{nm}$ are useful for cellular uptake and potentially the accumulation in tumor tissue, while larger particles in normal tissue would have slower uptake and tumor accumulation, perhaps due to passive targeting through an enhanced uptake permeability retention (EPR) effect. ${ }^{32}$ Recently, cellular internalization and blood circulation of PEGCPP-SA terpolymer, a polyanhydride based micelle of spherical, rod and comb (average mean diameter of the particle size between 300 to $400 \mathrm{~nm}$ ) shape regulated particles was shown. ${ }^{33}$

\section{UV-VIS spectrophotometric method validation}

The method for $\mathrm{DOX} \cdot \mathrm{HCl}$ was determined to be precise and accurate. The correlation coefficient $\left(R^{2}\right)$ was 0.9996 , the equation was $y=0.017 x+0.001$ with a slope $=0.017$ and the intercept was 0.001 . At the low drug concentration, the coefficient of variation $(\mathrm{CV} \%)$ was 2.6 and $2.8 \%$ when correlated to the highest drug concentration of 0.24 and $0.21 \%$.However, the percent accuracy at the lowest and highest drug concentrations was over $96.6 \%$. The limit of quantification (LOQ) and limit of detection (LOD) were established. ${ }^{21}$ We found the limit of detection and limit of quantification of the drug to be $1.62 \mathrm{mcg} / \mathrm{ml}$ and $0.87 \mathrm{mcg} / \mathrm{ml}$.

\section{DSC AND XRD analysis}

As illustrated in Figure 2, the sharp endothermic peaks of $\mathrm{DOX} \cdot \mathrm{HCl}(\mathrm{a})$, and blank nanoformulations (b) appeared

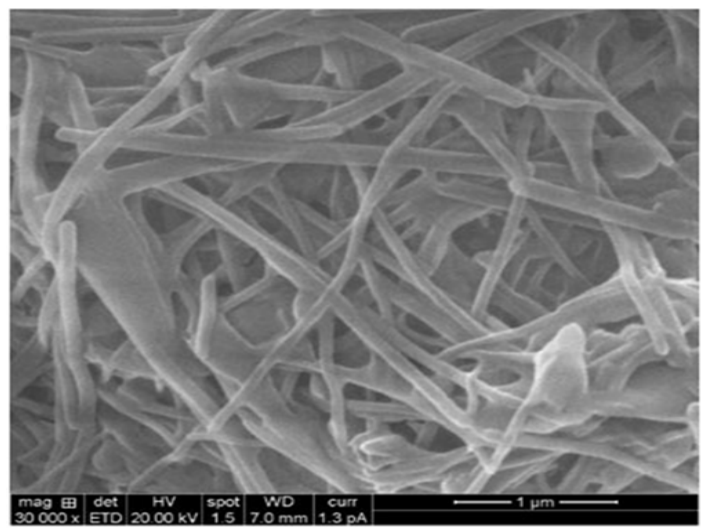

Figure 1: Typical SEM microphotographs of DOX $\cdot \mathrm{HCl}$ loaded $p((D L L A: C O) 60: 40)$ nanoformulation F-4 showing a cylindrical shape with a smooth surface. at $211.16,66.16^{\circ} \mathrm{C}$. We selected the blank nanocarrier as a control sample because the synthesized polymer has a rubbery nature and is difficult to assess by XRD. However, the drug peak disappearance in $\mathrm{DOX} \cdot \mathrm{HCl}$ loaded lyophilized nanoformulations F-1 to F-4. To analyze this event, we performed the XRD experiments. The X-ray diffraction patterns of the crystalline $\mathrm{DOX} \cdot \mathrm{HCl} 2 \theta$ scattered angle peaks performed at 22.27and $24.76^{\circ}$ with their conforming peak intensities of 1332 and 1378 (a). Blank nanoformulations showed 4.33 and $6.15^{\circ}$ peak intensities at 3589 and 2577 (b). Crystalline scattered angle peaks disappeared in the drug nanoformulations F-1 to F-4 (Figure 3) suggesting that the drug was present as a disordered crystalline or amorphous state. The nanoformulations maintained the crystalline cylinder shape of a smooth surface with $\mathrm{DOX} \cdot \mathrm{HCl}$.

\section{Drug release testing}

The in vitro release performance of the $\mathrm{DOX} \cdot \mathrm{HCl}$ and DOX $\cdot \mathrm{HCl}$ loaded P-(DLLA-CO:60:40) nanoformulations is illustrated in Figure 4. There was no first burst effect from the nanoformulations. This could be due to free $\mathrm{DOX} \cdot \mathrm{HCl}$ on the surface of the nanoformulations. The sink conditions were enhanced at intervals by the addition of new media every hour for 24 hrs. The effect of hydrophilic p(DL lactic acid) and hydrophobic (CO) on the release analysis of 5 and $10 \% \mathrm{w} / \mathrm{w}$ drug loaded F-1 and F-2 nanoformulations showed a prolonged pattern 80 and $86 \%$ of cumulative drug release was established. This delayed drug release may be due to increase in hydrophobic polymer carrier and in turn increases the hydrolytic degradation rate of the polymer. ${ }^{35}$ Another interpretation is that fatty acids may keep a drug encapsulated for a longer time when they need drug carriers. Castor oil is a triglyceride of recinolic acid. Castor oil content enhances the

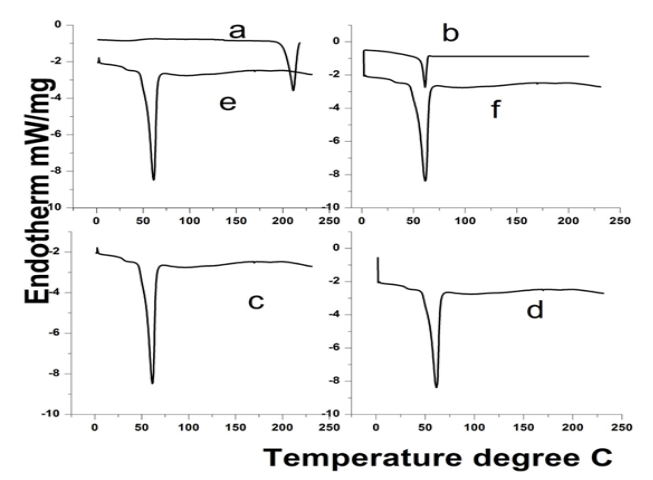

Figure 2: Sharp endothermic peaks detected by DSC studies of DOX. $\mathrm{HCl}(a)$, Blank-p(DLLA:CO) 60:40 nanoformulations (b), F-1 (c), F-2 (d), F-3 (e) and F-4 (f). 
hydrophobicity and retards the rate of hydrolytic degradation of recinolic acid (RA/triglyceride). However, as the concentration of $\mathrm{DOX} \cdot \mathrm{HCl}$ increased in the nanocarrier with the same amount of polymer availability, the amount of drug released is increased from drug-loaded nanoformulations of F3 (15\%) and F4 $(20 \%)$. It is possible that as the concentration of castor oil decreases the drug release will be faster. The drug release may be either faster or delayed depending on different chemical properties and physical, such as the crystallinity of the polymer, hydrophobicity of monomers and polymer, degradation medium, and water penetration into the nanoformulations. ${ }^{33-35}$ From the release kinetics data we concluded that the nanoformulations followed zero-order and Korsmeyer Peppas models (Table 3).

\section{In-vitro cytotoxicity test of nanoformulations}

The cytotoxic effect of $\mathrm{DOX} \cdot \mathrm{HCl}, \mathrm{DOX} \cdot \mathrm{HCl}$ loaded P-(DLLA-CO:60:40) nanoformulations and empty nanoformulations on MCF-7 breast cancer cell lines was evaluated. As seen in Figure 5, DOX $\cdot \mathrm{HCl}$ loaded

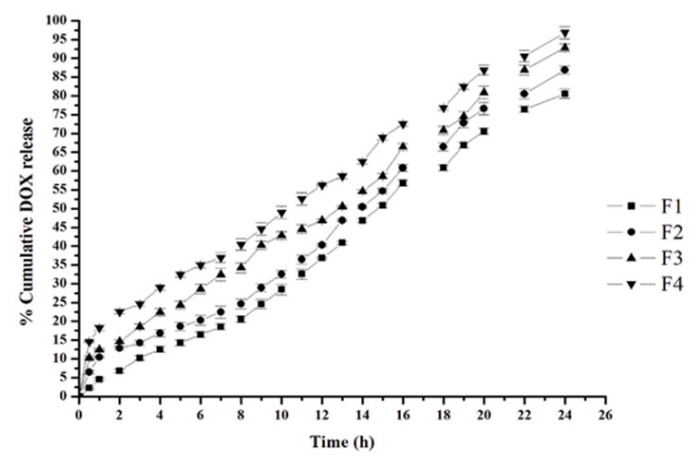

Figure. 4. The cumulative in vitro drug release of $\mathrm{DOX} \cdot \mathrm{HCl}$ loaded p(DLLA:CO) 60:40) nanoformulations F-1 ( $)$ ), F-2 ( $\square$ ), F-3 $(\Delta)$ and F-4 $(\bullet)$ shown as a percent at intervals over 24 hrs.
P-(DLLA-CO:60:40) nanoformulations decreased the survival of the MCF-7 breast cancer cells in a concentration-dependent manner similar to free

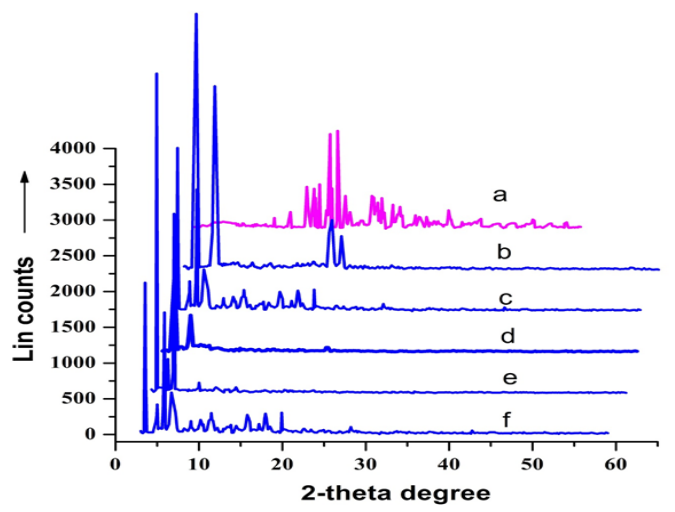

Figure. 3. XRD patterns of DOX.HCl (a), B1-p(DLLA:CO) 60:40 nanoformulations (b), F-1 (c), F-2 (d), F-3 (e) and F-4 (f). Crystalline scattered angle peaks disappeared in the drug nanoformulations F-1 to F-4 suggesting that the drug was present as a disordered crystalline or amorphous state.

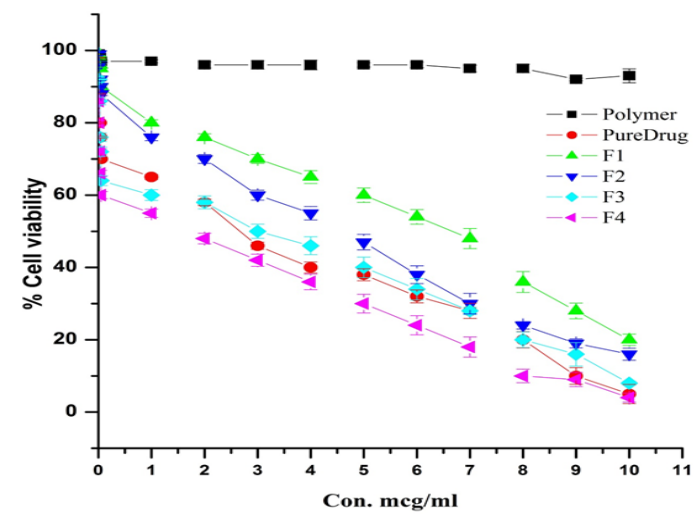

Figure. 5. Inhibition of MCF-7 cells after incubation with blank nanoformulations ( $\bullet$ ), DOX·HCl $(\Delta), \mathrm{F}-1(\bullet), \mathrm{F}-2(\nabla)$, F-3 (४), and F-4 (४). The cell viability of MCF-7 breast cancer cell lines after $24 \mathrm{hrs}$ of incubation with concentrations from 0.001 to $10 \mathrm{mcg} / \mathrm{mL}$ of $\mathrm{DOX} \cdot \mathrm{HCl}$ and blank nanoformulations was measured with an MTT assay. Inhibition was concentration-dependent.

Table 3. Release rate kinetics of DOXHCI loaded p(DLLA:CO) 60:40 nanoformulations.

\begin{tabular}{|c|c|c|c|c|c|}
\hline Release Mechanisms & Values & F-1 & F-2 & $F-3$ & F-4 \\
\hline \multirow{2}{*}{$\begin{array}{l}\text { Zero order } \\
Q_{t=} Q_{0}+K_{0} t\end{array}$} & $\mathrm{R}^{2}$ & 0.968 & 0.967 & 0.969 & 0.974 \\
\hline & Slope & -0.261 & -0.264 & -0.257 & -0.267 \\
\hline \multirow{2}{*}{$\begin{array}{c}\text { First order } \\
\text { In } Q_{t=} \ln Q_{0}+K_{0} t\end{array}$} & $\mathrm{R}^{2}$ & 0.949 & 0.956 & 0.961 & 0.971 \\
\hline & Slope & -0.063 & -0.063 & -0.058 & -0.071 \\
\hline \multirow{2}{*}{$\begin{array}{c}\text { Matrix } \\
\text { (Higuchi Matrix) } \\
\mathrm{Q}_{\mathrm{t}=} \mathrm{K}_{\mathrm{H}} \text { sq.t }\end{array}$} & $\mathrm{R}^{2}$ & 0.947 & 0.945 & 0.947 & 0.930 \\
\hline & Slope & 1.102 & 1.126 & 1.081 & 1.167 \\
\hline \multirow{3}{*}{$\begin{array}{l}\text { Korsmeyer- } \\
\text { Peppas } \\
Q_{t} / Q_{\text {inf }} K_{k} t^{n}\end{array}$} & $\mathrm{R}^{2}$ & 0.959 & 0.941 & 0.962 & 0.966 \\
\hline & Slope & 0.263 & 0.276 & 0.279 & 0.257 \\
\hline & $\mathrm{n}$ & 0.273 & 0.273 & 0.281 & 0.266 \\
\hline
\end{tabular}


DOX $\cdot \mathrm{HCl}$. The $\mathrm{IC}_{50}$ values of nanoformulations $\mathrm{F}-1$ and F-2 were $4.8 \pm 0.06$ and $3.9 \pm 0.07 \mathrm{mcg} / \mathrm{ml}$. The $\mathrm{IC}_{50}$ values ofF3 and $\mathrm{F} 4$ were $3.2 \pm 0.03$ and1.98 \pm 0.08 $\mathrm{mcg} / \mathrm{ml}$. The $\mathrm{IC}_{50}$ for inhibition by the free drug was $2.2 \mathrm{mcg} / \mathrm{ml}$. These data show that drug release from nanoformulations was concentration-dependent and related to the amount of drug loading in the nanoformulations. The development of drug aggregates and degradation of a large amount of polymer might limit its movement into cells (in the case of formulation F-1 and F-2). The action of F-3 and F-4 formulations with lower inhibition and $\mathrm{IC}_{50}$ values may have faster hydrolytic degradation or erosion of the nanoformulations and a greater variation in the drug loading and the copolymer Poloxamer-F127 in the nanoformulations. Based on the cellular viability studies, DOX-loaded nanoformulations readily release the drug into the cells. We concluded there was a higher level and faster drug release with more permeability of drug. The present result is consistent with published findings. ${ }^{1,10} \mathrm{DOX} \cdot \mathrm{HCl}$ nanoformulations showed a significant cytotoxic effect through the controlled release of encapsulated drug from the polymer, verifying that $\mathrm{DOX} \cdot \mathrm{HCl}$ encapsulated $\mathrm{F} 127$ is active. Others showed that F127 directly inhibits drug efflux pumps by incorporating into cells and influencing, ATP synthesis, mitochondrial respiration, and the efflux activity. ${ }^{10,35}$ Also, the architecture, cylindrical rod shape, the geometry and surface aspects of these nanoformulations may influence the effects on MCF-7 breast cancer cell lines. ${ }^{6}$

\section{CONCLUSION}

We prepared DOX $\cdot \mathrm{HCl}$ loaded P-(DLLA-CO:60:40) nanoformulations with a copolymer of F127 by a solvent evaporation thin-film hydration method. The concept related to the use of this polymer was to reduce hydrolysis and to generate a more lipophilic carrier, which could increase the degradation time of the polymer and influence the $\mathrm{DOX} \cdot \mathrm{HCl}$ release time and efficacy in nanoformulations. This method produces a high yield of nanoformulations as well as drug loading and encapsulation efficiency. F127 was used as a copolymer/stabilizer. F127 is a non-ionic co-emulsifier added during the preparation of nanoformulations and its use results in high drug loading. This polymer may have a role in generating cylindrical rod shapes and smaller particle size with narrow size distribution and its more effective activity on MCF-7 cell lines. The experiment carried out on DSC and XRD studies revealed that P-(DLLA-CO:60:40) nanoformulations encapsulated drug was in the form disorder crystalline or amorphous state. The in vitro release behavior of the DOX $\cdot \mathrm{HCl}$ loaded P-(DLLA-CO:60:40) nanoformulations showed there was no initial burst effect from the drug-loaded nanoformulations. The drug release depends on the drug-loading concentration of the DOX $\cdot \mathrm{HCl}$ in the nanoformulations. Delayed controlled drug release for $24 \mathrm{hrs}$ was determined and release kinetics followed zero-order and Korsmeyer Peppas models. The drug-loaded nanoformulations retained their biological effect as evidenced by a significant cytotoxic effect on MCF-7 breast cancer cell lines. The current investigation of $\mathrm{DOX} \cdot \mathrm{HCl}$ loaded P-(DLLA-CO:60:40) nanoformulations with a copolymer of F127 by solvent evaporation film hydration method demonstrates a potential drug delivery system for cancer.

\section{Data Availability}

All supporting data (SEM, DSC, IR, XRD, UV Spectrophotometric, In vitro percentage drug release, in vitro cytotoxicity,) analyzed during this research work are included within the article.

\section{Authors' Contributions}

All authors performed experiment, performed analysis, interpreted results, writing, and revising the paper, gave approval for the final version submitted for publication. Agree to be accountable for all aspects of this work.

\section{ACKNOWLEDGEMENT}

The authors are thankful to the Deanship of Scientific Research, King Faisal University, Al-Ahsa for financial support under Nasher track (Grant No. 186198).

\section{CONFLICT OF INTEREST}

All the authors declare that there are no conflicts of interest regarding the publication of this research paper.

\section{ABBREVIATIONS}

IAV: Influenza A Virus; PHB: Poly(hydroxybutyrate, PSA: Polysebecic anhydride; PLA: Polyesters of Poly (lactic acid); PLA: Poly(caprolactone); CO: Castor oil; DOX:HCL: Doxorubicin hydrochloride PBS: Phosphate-buffered saline; XRD: X-ray diffraction; DSC: Differential scanning calorimeter

\section{REFERENCES}

1. Amiri Z, Moghadam MF, Sadeghizadeh M. Anticancer Effects of DoxorubicinLoaded Micelle on MCF-7 and MDA-MB-231, Breast Cancer Cell Lines. Journal Research Medical Dental Science. 2018;6(2):298-304. 
2. Bodratti AM, Alexandridis P. Formulation of Poloxamers for Drug Delivery. Journal Functional Biomaterial. 2018;9(1):11.

3. Dhakal S, Goodman J, Bondra K, Lakshmanappa YS, Hiremath J, Shyu DL. Polyanhydride nanovaccine against swine influenza virus in pigs. Vaccine. 2107;35(8):1124-31.

4. Domb A, Langer R. Polyanhydrides. I. Preparation of high molecular weight polyanhydrides. Journal of Polymer Science Part A: Polymer Chemistry. 1987;25(12): 3373-86.

5. Domb AJ, Amselem S, Shah J, Maniar M. Polyanhydrides: synthesis and characterization. Biopolymers. 1993;93-141.

6. Yang G, Wang J, Li D, Zhou S. Polyanhydride micelles with diverse morphologies for shape-regulated cellular internalization and blood circulation. Regenration Biomaterial. 2017;4(3):149-57.

7. Doppalapudi S, Jain A, Khan W, Domb AJ. Biodegradable polymers-an overview. Polymers for Advanced Technologies. 2014;25(5):427-35.

8. Kumar N, Ravikumar MN, Domb AJ. Biodegradable block copolymers. Adv Drug Deliv Rev. 2001;53(1):23-44.

9. Kunduru KR, Basu A, Haim Zada M, Domb AJ. Castor Oil-Based Biodegradable Polyesters. Biomacromolecules. 2015;16(9):2572-87.

10. Gao Z, Zhang L, Sun Y. Nanotechnology applied to overcome tumor drug resistance. Journal of Control Release. 2012;162(1):45-55.

11. Heller J, Barr J. Poly(ortho esters) from concept to reality. Biomacromolecules. 2004,5(5),1625-1632.

12. Heller J, Barr J, Ng S, Shen H, Schwach-Abdellaoui K, Emmahl S. Poly (ortho esters)-their development and some recent applications. European Journal of Pharmaceutics and Biopharmaceutics. 2000;50(1):121-8.

13. Heller J, Barr J, Ng SY, Abdellauoi KS, Gurny R. Poly(ortho esters): synthesis, characterization, properties and uses. Advances in Drug Delivery Review. 2002;54(7):1015-1039.

14. Hiremath J, Kusum Devi V, Devi K, Domb A. Biodegradable poly (sebacic acid-co-ricinoleic-ester anhydride) tamoxifen citrate implants: Preparation and in vitro characterization. Journal of Applied Polymer Science. 2008;107(5):2745-54.

15. Hiremath JG, Rajeshkumar A, Ickowicz D, Domb AJ. Preparation and in vitro characterization of paclitaxel containing poly(lactic acid co-castor oil)based nanodispersions. Journal of Drug Delivery Science and Technology. 2013;23(5):439-44.

16. Hiremath J, Rudani C, Domb A, Suthar R, Khamar N. Preparation and in vitro characterization of poly (sebacic acid-co-ricinoleic acid)-based tamoxifen citrate-loaded microparticles for breast cancer. Journal of Applied Polymer Science. 2012;124(6):4747-54.

17. Hiremath JG, Rudani CG, Suthar RV, Domb AJ. Tamoxifen citrate loaded biodegradable poly(sebacic acid-co-ricinoleic acid) microparticles, in vitro characterization. J Drug Delivery Science Technology. 2011;21(5):417-22.

18. Duchene D, Gref R. Small is beautiful: Surprising nanoparticles. International Journal Pharmaceutics. 2016;502(1-2):219-31.

19. Minotti G, Menna P, Salvatorelli E, Cairo G, Gianni L. Anthracyclines: molecular advances and pharmacologic developments in antitumor activity and cardiotoxicity. Pharmacol Rev. 2004;56(2):185-229.

20. Prados J, Melguizo C, Ortiz R, Velez C, Alvarez PJ, Arias JL. Doxorubicinloaded nanoparticles: new advances in breast cancer therapy. Anticancer Agents Medicinal Chemistry. 2012;12(9):1058-70.
21. PrashanthKumar R, Hiremath JG. In vitro physicochemical characterisation of doxorubicin-loaded poly ( $\varepsilon$-caprolactone). International Journal of Nano and Biomaterials. 2016;6(2):63-73.

22. Santander-Ortega MJ, Jodar-Reyes AB, Csaba N, Bastos-Gonzalez D, Ortega-Vinuesa JL. Colloidal stability of pluronic F68-coated PLGA nanoparticles: a variety of stabilisation mechanisms. J Colloid Interface Science. 2006;302(2):522-529.

23. Yan F, Zhang C, Zheng Y, Mei L, Tang L, Song C. The effect of poloxamer 188 on nanoparticle morphology, size, cancer cell uptake, and cytotoxicity. Nanomedicine, 2010,6(1),170-178.

24. Hoo CM, Starostin N, West P, Mecartney ML. A comparison of atomic force microscopy (AFM) and dynamic light scattering (DLS) methods to characterize nanoparticle size distributions. Journal Nanoparticle Research. 2008;10(1):89-96.

25. SreeHarsha N, Hiremath JG, Aitha RK, Domb AJ, Al-Dhubiab BE, Ramnarayanan C. Paclitaxel loaded poly (DL lactic acid co castor oil) 60: 40 with poloxamer-F68 rod shape cylindrical nanoparticle preparation and in vitro cytotoxicity studies. Polymers for Advanced Technologies. 2019;30:2613-22.

26. Denizot F, Lang R. Rapid colorimetric assay for cell growth and survival. Modifications to the tetrazolium dye procedure giving improved sensitivity and reliability. Journal Immunololgy Methods. 1996;89(2):271-7.

27. Hong W, Chen DW, Zhao XL, Qiao MX, Hu HY. Preparation and study in vitro of long-circulating nanoliposomes of curcumin. China Journal of Chinese Materia Medica, 2008;33(8):889-92.

28. Sharma N, Madan P, Lin S. Effect of process and formulation variables on the preparation of parenteral paclitaxel-loaded biodegradable polymeric nanoparticles: A co-surfactant study. Asian Journal of Pharmaceutical Sciences. 2016;11(3):404-416.

29. Ai X, Zhong L, Niu H, He Z. Thin-film hydration preparation method and stability test of DOX-loaded disulfide-linked polyethylene glycol 5000-lysinedi-tocopherol succinate nanomicelles. Asian Journal of Pharmaceutical Sciences. 2014;9(5):244-50.

30. Golombek SK, May J-N, Theek B, Appold L, Drude N, Kiessling F. Tumor targeting via EPR: Strategies to enhance patient responses. Advanced Drug Delivery Reviews. 2018;130:17-38.

31. Budhian A, Siegel SJ, Winey KI. Production of haloperidol-loaded PLGA nanoparticles for extended controlled drug release of haloperidol. Journal Microencapsulation. 2005;22(7):773-85.

32. Sokolsky-Papkov M, Golovanevski L, Domb AJ, Weiniger CF. Prolonged local anesthetic action through slow release from Poly(Lactic Acid Co Castor Oil). Pharmaceutical Research. 2008;26(1):32.

33. Hiremath JG, Kusum Devi V, Devi K, Domb AJ. Biodegradable poly(sebacic acid-co-ricinoleic-ester anhydride) tamoxifen citrate implants: Preparation and in vitro characterization. Journal of Applied Polymer Science. 2008;107(5):2745-54.

34. Sokolsky-Papkov M, Domb AJ. Stereoisomeric effect on in vitro drug release from injectable poly(lactic acid co castor oil) polyesters. Polymers for Advanced Technologies. 2008;19(6):671-9.

35. Yan F, Zhang C, Zheng Y, Mei L, Tang L, Song C. The effect of poloxamer 188 on nanoparticle morphology, size, cancer cell uptake, and cytotoxicity. Nanomedicine Nanotechnol Biololgy Medicine. 2010;6(1):170-8. 
PICTORIAL ABSTRACT

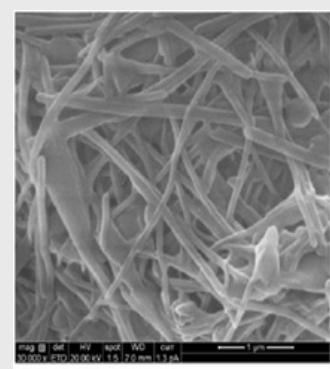

DOX $\cdot \mathrm{HCl}$ loaded $\mathrm{p}((\mathrm{DLLA}: \mathrm{CO})$ 60:40) nanoformulation

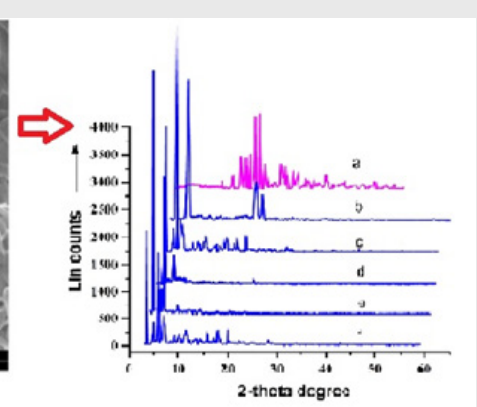

XRD patterns of DOX $\cdot \mathrm{HCl}$

\section{SUMMARY}

The present research endeavor is to prepare and characterize $p(D L L A: C O)$ (60:40) nanosized particles containing DOX. DOX loaded p(DLLA:CO) (60:40) nanosized particles were well processed by nanoprecipitation method. Hence, sustained/controlled release phenomenon was obtained in developed formulations when subjected for in-vitro release study. The nano formualtion was performed in vitro cytotoxicity studies in MCF-7 cell culture. The action of F-3 and F-4 formulations with lower inhibition and $\mathrm{IC}_{50}$ values may have faster hydrolytic degradation or erosion of the nanoformulations and a greater variation in the drug loading and the copolymer Poloxamer-F127 in the nanoformulations.

\section{About Authors}

Dr. Sree Harsha received his (ranked top 5) Master of Pharmacy Degree and subsequently earned a doctorate in Pharmaceutics from Rajiv Gandhi University of Health Sciences, Bangalore, India in 2006. He came to King Faisal University in 2007 as an assistant professor in the Department of Pharmaceutical Sciences, bringing with him several years' worth of teaching experience in fundamentals of pharmaceutics and drug delivery systems. He was actively participated in Accreditation Council of Pharmacy Education (ACPE) and Canadian Council for the Accreditation of Pharmacy Programs (CCAPP). His primary area of focus is pharmaceutical technology and novel/ targeted drug delivery systems. For this research, he received grants (30 number) from Deanship of Scientific research, King Faisal University. The author contributed so far to 85 peer-reviewed full papers on a variety of topics in lung targeting, topical drug delivery and mucoadhesive drug delivery systems, He has contributed in writing a book chapter titled "Targeted Drug Delivery System" and "Microspheres" in Textbook of Industrial Pharmacy, Publisher-Orient Longman Private Ltd. In addition, he is an Ad-hoc reviewer for scientific journals. He has attended many seminars and Workshop both national and international on Pharmaceutical Technology and Public health issues.

Cite this article: Sreeharsha N, Hiremath JG, Kumar PR, Meravanige G, Khan S, Karnati RK, et al. Doxorubicin Hydrochloride Loaded Polyanhydride Nanoformulations And Cytotoxicity. Indian J of Pharmaceutical Education and Research. 2021;55(1):117-25. 